\title{
Spontaneous fragmentation and embolization of chemoport catheter- a case report
}

\section{Introduction}

Central venous access devices are commonly utilized in cancer patients. These devices include central venous catheters, peripherally inserted central catheter (PICC line), Hickman catheter and totally implantable central venous devices of the port-a-cath type (chemoport). These devices can be retained for a long time and may be used for administration of chemotherapy, blood products, total parenteral nutrition and frequent blood sampling. Complications associated with chemoport include vascular injury, hemothorax, pneumothorax, local site infection, sepsis, catheter thrombosis, drug extravasation and mechanical malfunction. Spontaneous fragmentation of chemoport catheter is a rare complication. We report a case of spontaneous fragmentation of chemoport catheter from its midway and its migration into the lower lobar branch of left pulmonary artery. It was retrieved using percutaneous endovascular approach under fluoroscopic guidance.

\section{Case report}

A twenty nine years old lady diagnosed as Carcinoma left breast underwent breast conservation surgery (pT2N2aM0, ER positive PR positive Her 2 neu positive) and presented for adjuvant chemotherapy. She was planned for adjuvant chemotherapy, radiotherapy and hormonal therapy as per standard guidelines. Chemoport reservoir was placed in the chest wall - right infraclavicular fossa and chemoport catheter was placed in the right subclavian vein. Postprocedure chest $\mathrm{x}$ ray revealed proper placement of the chemoport. She underwent first cycle of adjuvant chemotherapy with Docetaxel, Carboplatin and Trastuzumab without any complications. When she presented for second cycle (45 days after chemoport insertion), she was asymptomatic. However, there was no backflow of blood from the chemoport reservoir. Chest $\mathrm{X}$ ray revealed fragmentation and embolization of chemoport catheter. ECG showed normal sinus rhythm. Non Contrast CT scan chest was done, which revealed that the distal fragment of chemoport catheter had embolized to left pulmonary artery lower lobar branch, having migrated across the right atrium, right ventricle, main pulmonary artery and its left branch (Figure 1). After informed consent, retrieval of distal fragment of chemoport catheter was done in cath lab under local anesthesia under fluoroscopic guidance by Interventional Radiology team. It was retrieved from left pulmonary artery lower lobar branch in a procedure lasting forty minutes. The right femoral vein was punctured, $10 \mathrm{Fr}$ Cordis sheath was introduced into the femoral vein, left pulmonary artery was cannulated using cobra 5 Fr catheter and then it was exchanged with snare over the guide wire. The left posterior inferior branch of pulmonary artery was selectively cannulated using Terumo guide wire and Cobra catheter. The tip of the foreign body was snared using Sequre snare system $(4 \mathrm{Fr}, 10 \mathrm{~mm})$ and pulled through the pulmonary artery (Figure 2). The length of the catheter fragment was $4 \mathrm{~cm}$ (Figure 3-9). The next day, chemoport reservoir and the proximal fragment of chemoport catheter was retrieved by surgery under local anesthesia. Patient tolerated both procedures well and was discharged on the third day.
Volume II Issue 6 - 202I

\author{
Vibhor Sharma,' Chandra Prakash Singh \\ Chauhan, ${ }^{2}$ Dhirendra Pratap Singh, ${ }^{3}$ Pawan \\ Gupta $^{4}$ \\ 'Department of Medical Oncology and Bone Marrow \\ Transplantation, Paras Hospital, India \\ ${ }^{2}$ Department of Radiology and Interventional Radiology, Jaypee \\ Hospital, India \\ ${ }^{3}$ Department of Radiology, Jaypee Hospital, India \\ ${ }^{4}$ Department of Surgical Oncology, Max Hospital, India
}

Correspondence: Vibhor Sharma, Department of Medical Oncology and Bone Marrow Transplantation, Paras Hospital, India,Tel+91773851971 I, Email vibhor81@yahoo.co.in

Received: July 06, 202। | Published: November 22, 202 |

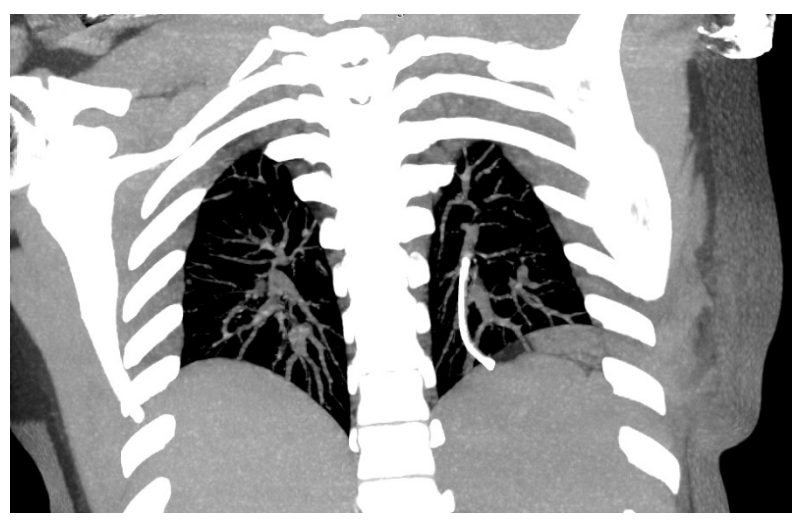

Figure I Magnified Intensity Projection (MIP) image coronal view NCCT Chest showing distal fragment of chemoport catheter in the left pulmonary artery lower lobar branch.

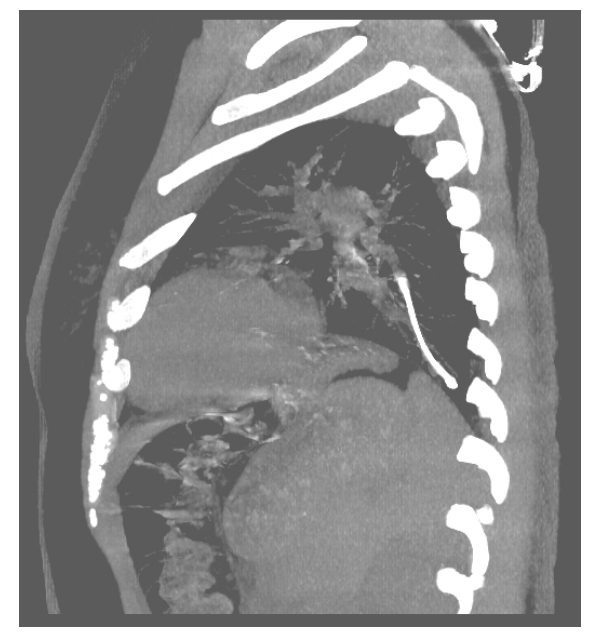

Figure 2 MIP image sagittal view NCCT Chest showing distal fragment of chemoport catheter in the left pulmonary artery lower lobar branch. 


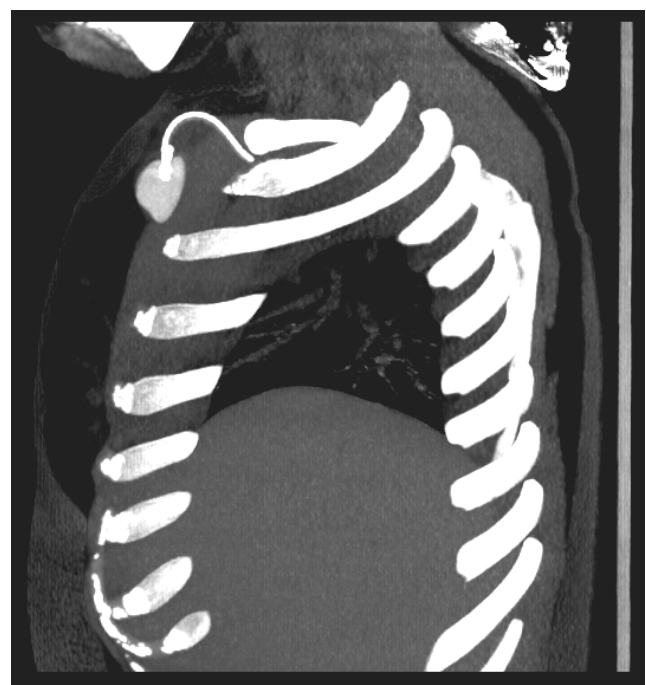

Figure 3 MIP image sagittal view NCCT Chest showing chemoport reservoir in the chest wall.

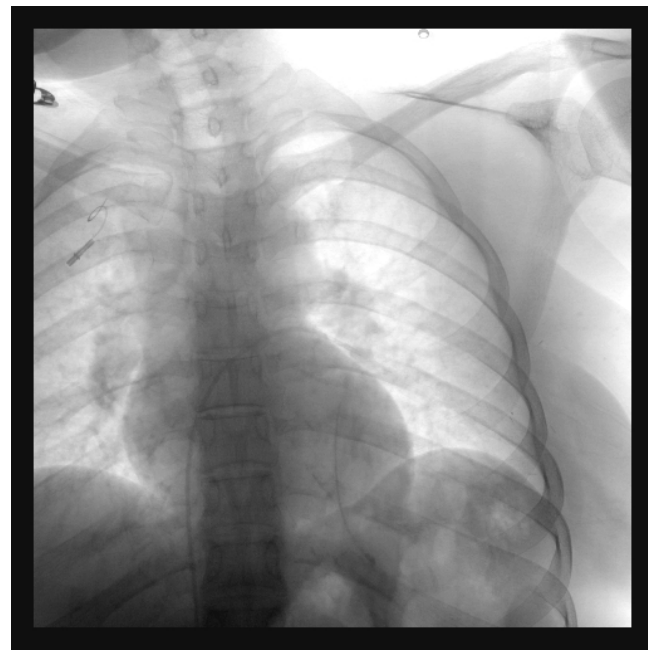

Figure 4 Fluoroscopy image showing snare catheter in left pulmonary artery with foreign body in left pulmonary artery branch.

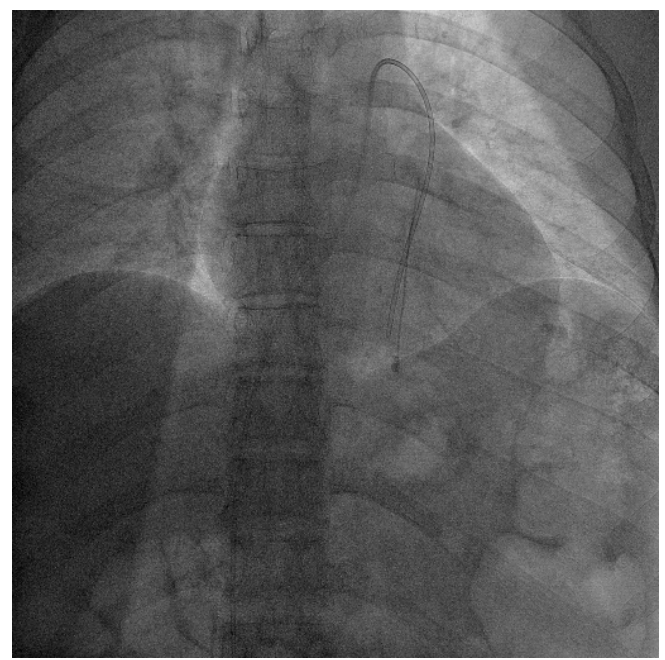

Figure 5 Fluoroscopy image showing snare catheter adjacent to the foreign body in left pulmonary artery branch.

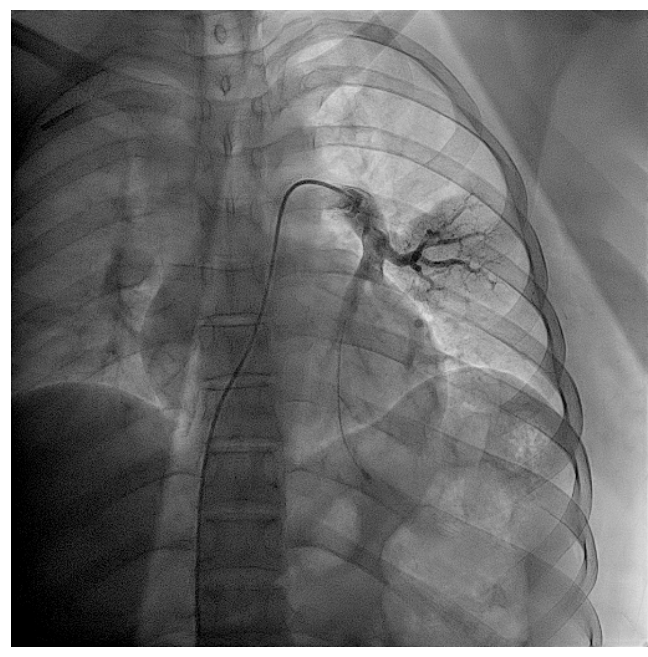

Figure 6 Fluoroscopy image of Catheter pulmonary angiogram showing the foreign body in left pulmonary artery branch.

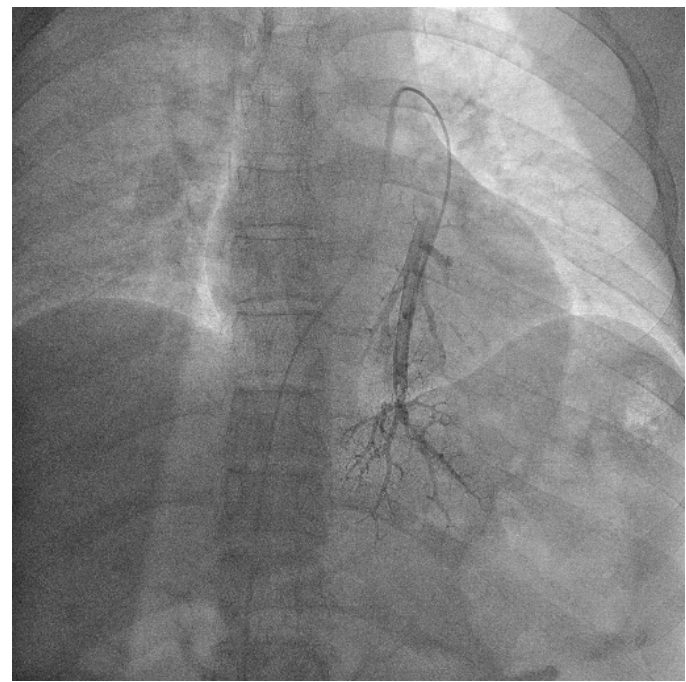

Figure 7 Fluoroscopy image of Catheter pulmonary angiogram showing foreign body as a filling defect in the lower lobar branch of left pulmonary artery.

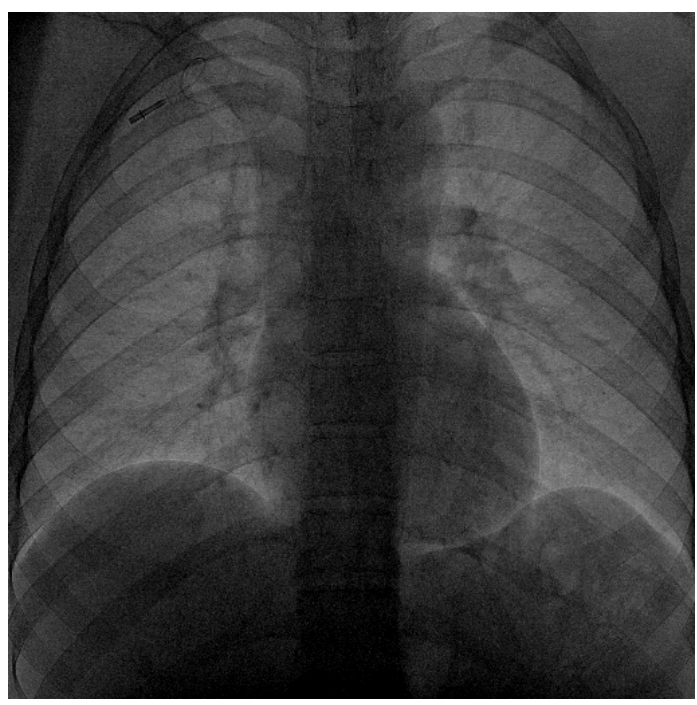

Figure 8 Check fluoroscopy image after removal of foreign body. 


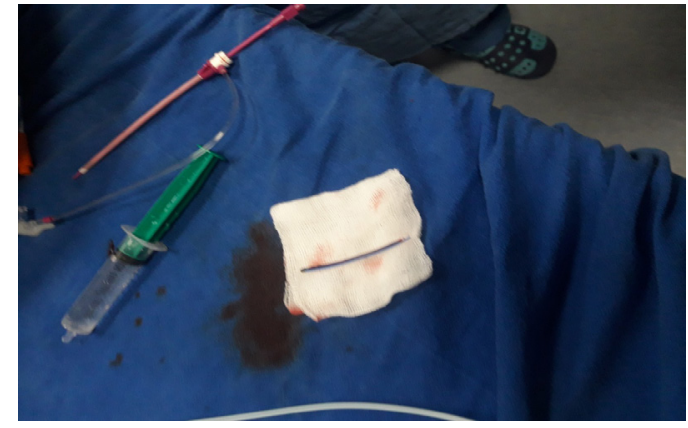

Figure 9 Distal fragment of the chemoport catheter retrieved.

\section{Comment}

Spontaneous rupture of the chemoport catheter is a rare but lifethreatening complication. The incidence of chemoport fracture reported in recent series is $0.4 \%$ to $1.8 \% .^{1,2}$ These patients may be asymptomatic or may experience symptoms like palpitations, chest discomfort, paresthesias in the arm, breathlessness, swelling and resistance to infusion. Blood aspiration followed by heparinized saline injection should be routinely performed before chemotherapy infusion through chemoport. Inability to aspirate blood or local pain or subcutaneous swelling during saline flushing should alert the physician regarding the possibility of chemoport fracture.

Contributory factors implicated for chemoport fracture include site of port implantation, material of chemoport catheter, improper fixation of chemoport catheter to the locking steel ring or repeated high pressure injections to resolve clot formation.

A subclavian "pinch off" syndrome has been described in relation to chest wall implantation of chemoport. ${ }^{3}$ Rupture of the catheter most frequently occurs in the costoclavicular space - between the first rib and the clavicle. At this site, the catheter is constantly subjected to a pincer movement between the clavicle and the first rib. Subclavian "pinch off" syndrome may be prevented by using the internal jugular vein approach or a more lateral approach through the subclavian vein.

The most common site of chemoport fracture is at the anastomosis between the injection port and the catheter in $93.2 \%$ of cases followed by the middle part in $6.8 \% .{ }^{4}$ Among various catheter materials, higher resistance of silicone catheters has resulted in less frequent rupture as compared to polyurethane catheters. ${ }^{5}$

The catheter fragment may embolize to the vena cava, right atrium, right ventricle, pulmonary artery or internal jugular vein. The most common site of embolization of fractured fragment is between the superior vena cava and the right atrium. ${ }^{6}$

In our patient, no specific cause of catheter fragmentation could be identified. The fragmentation took place through the middle of the catheter. The catheter fragment embolized to the left pulmonary artery lower lobar branch.

Any fragment of the chemoport catheter must be removed early. This is best done using percutaneous endovascular techniques under fluoroscopic guidance with specific catheters and snare loops. If these techniques fail, open surgery should be considered.

\section{Acknowledgments and disclosures}

I declare that no undisclosed authors have contributed to this work. I declare that consent has been taken from the patient regarding the publication of this manuscript for scientific purpose.

\section{Conflicts of interest}

The authors of this manuscript declare no conflicts of interest.

\section{Source of funding}

We did not receive any funds from any sources during the preparation of this manuscript.

\section{References}

1. Filippou D, Tsikkinis C, Filippou GK, et al. Rupture of totally implantable central venous access devices (intraports) in patients with cancer: report of four cases. World J Surg Oncol. 2004;2:36.

2. Kamayama H, Yamazaki T, Maeda C, et al. Central venous access port device (CVAPD) related complications in colorectal cancer patients. Gan To Kagaka Ryoho. 2010;37:453-455.

3. Mirza B, Vanek VW, Kupensky DT: Pinch-off syndrome: case report and collective review of the literature. Am Surg. 2004;70: 635-644.

4. Lin $\mathrm{CH}, \mathrm{Wu} \mathrm{HS}$, Chan DC, et al. The mechanism of failure of totally implantable central venous access system: Analysis of 73 cases with fracture of catheter. EJSO. 2010;36:100-103.

5. Vandoni RE, Guerra A, Sanna P, et al. Randomized comparison of complications from three different permanent central venous access systems. Swiss Med Wkly. 2009;130:313-316.

6. Cheng EC, Tsai TN, Yang EC, et al. Percutaneous removal of dislodged totally implantable central venous access system in 92 cases: Experience in a single hospital. Eur J Radiol. 2009;69:346-350. 